\title{
MATERNAL COMPLICATIONS OF SEVERE PREECLAMPSIA IN A LEVEL III UNIVERSITY HOSPITAL
}

\author{
García García CR, Guasch Arévalo E, Sancho de Ávila A, Schiraldi R, Gilsanz Rodríguez F.
} La Paz University Hospital, Department of Anaesthesiology and Intensive Care, Madrid, Spain.

\section{BACKGROUND}

In developed countries, the incidence of many risk factors of preeclampsia has increased: maternal age $\geq 35$, obesity, chronic hypertension, assisted reproductive technology and multiple gestation. This fact may have influenced the prognosis of severe preeclampsia $(\mathrm{SP})^{1}$.

\section{GOAL OF STUDY}

To describe maternal complications of SP in a level III University Hospital.

\section{MATERIAL AND METHODS}

Prospective observational study of women with $\mathrm{SP}^{2}$ admitted to a high dependency unit, from 2010 to 2012.

Table 1. Maternal complications analyzed
1. Obstetric
5. "Placental
haemorrhage $(\mathrm{OH})^{3}$ abruption"
2. Renal dysfunction
$(\mathrm{Cr}>1,2 \mathrm{mg} / \mathrm{dl})$
6. Acute pulmonary edema (APE)
3. Hepatopathy
7. Perinatal death
(AST/ALT $\geq 70 \mathrm{UI} / \mathrm{L}$ )
(20 weeks gestation- $7^{\text {th }}$ day life)
4. HELLP syndrome
8. Eclampsia

\section{RESULTS $(n=276)$}

Figure 1. Maternal complications

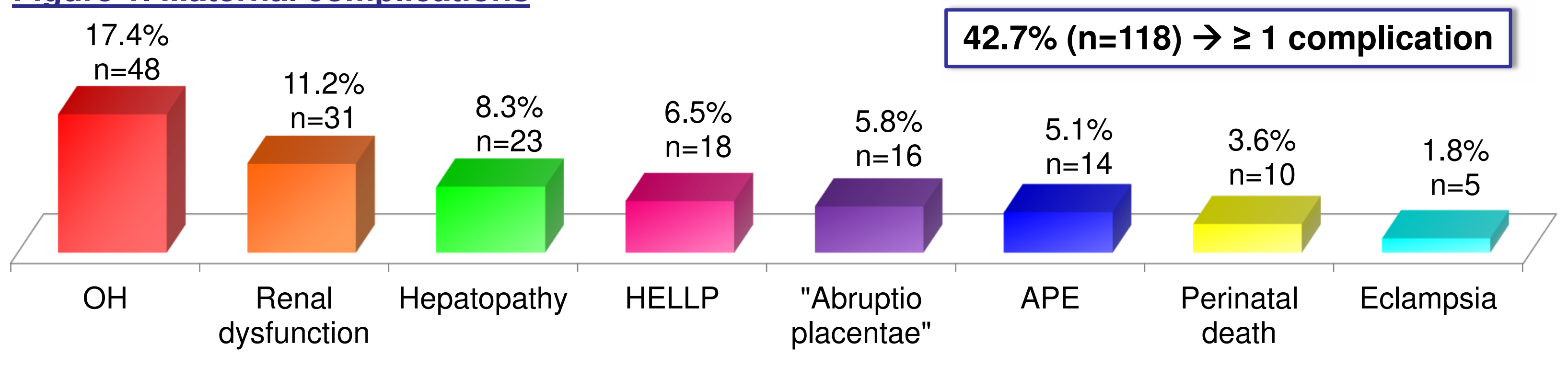

Fiqure 2. Risk factors of preeclampsia

\begin{tabular}{|l|l|}
\hline $44.6 \%(n=123)$ & Maternal age $\geq 35$ \\
\hline $17.3 \%(n=47)$ & Obesity (BMI $\geq 35 \mathrm{~kg} / \mathrm{m} 2)$ \\
\hline $16.7 \%(n=46)$ & Multiple gestation \\
\hline $16.3 \%(n=45)$ & Assisted reproductive technology \\
\hline $8.7 \%(n=24)$ & Chronic hypertension \\
\hline
\end{tabular}

Table 2. Factors associated to higher incidence of perinatal death

\begin{tabular}{|c|c|c|}
\hline & & $\begin{array}{c}\text { Perinatal death } \\
\text { n (\%) }\end{array}$ \\
\hline $\begin{array}{l}\text { Chronic hypertension } \\
(p=0.046)\end{array}$ & $\begin{array}{l}\text { No } \\
\text { Yes }\end{array}$ & $\begin{array}{l}7(2.8) \\
3(12.5)\end{array}$ \\
\hline $\begin{array}{l}\text { Early-onset preeclampsia } \\
(p<0.001)\end{array}$ & $\begin{array}{l}\text { No } \\
\text { Yes }\end{array}$ & $\begin{array}{l}1(0.5 \%) \\
9(10.8)\end{array}$ \\
\hline $\begin{array}{l}\text { Patological uterine } \\
\text { Doppler }(p<0.001)\end{array}$ & $\begin{array}{l}\text { No } \\
\text { Yes }\end{array}$ & $\begin{array}{c}0(0) \\
6(10.5)\end{array}$ \\
\hline
\end{tabular}

\section{DISCUSSION}

There was a higher incidence of $\mathrm{OH}$ and APE than described in literature, probably related to the prospective design, as well as the high percentage of multiple pregnancies and early-onset forms $(30.1 \%, \mathrm{n}=83)$.

\section{CONCLUSIONS}

The most frequent complications were $\mathrm{OH}$ and renal dysfunction. Early-onset preeclampsia was associated to a higher perinatal death rate.

\section{References:}

1. Knight M, Kenyon S, Brocklehurst P, Neilson J, Shakespeare J, Kurinczuk JJ (Eds.) on behalf of MBRRACEUK. Confidential Enquiries into Maternal Deaths and Morbidity 2009-12. Oxford: National Perinatal Epidemiology Unit, University of Oxford 2014.

2. Steegers EA, von Dadelszen P, Duvekot JJ, et al. Lancet. 2010; 376: 631-44.

3. ACOG. Obstet Gynecol. 2006;108:1039-47. 